\title{
Applying Novel Time-Frequency Moments Singular Value Decomposition Method and Artificial Neural Networks for Ballistocardiography
}

\author{
Alireza Akhbardeh, ${ }^{1}$ Sakari Junnila, ${ }^{1}$ Mikko Koivuluoma, ${ }^{1}$ Teemu Koivistoinen, $^{2}$ and Alpo Värri ${ }^{1}$ \\ ${ }^{1}$ Institute of Signal processing, Tampere University of Technology, Korkeakoulunkatu 1, 33101 Tampere, Finland \\ ${ }^{2}$ Department of Clinical Physiology and Nuclear Medicine, Tampere University Hospital, Teiskontie 35, \\ 33521 Tampere, Finland
}

Received 8 April 2005; Revised 5 April 2006; Accepted 10 September 2006

Recommended by Bernard Mulgrew

\begin{abstract}
As we know, singular value decomposition (SVD) is designed for computing singular values (SVs) of a matrix. Then, if it is used for finding SVs of an $m$-by-1 or 1-by- $m$ array with elements representing samples of a signal, it will return only one singular value that is not enough to express the whole signal. To overcome this problem, we designed a new kind of the feature extraction method which we call "time-frequency moments singular value decomposition (TFM-SVD)." In this new method, we use statistical features of time series as well as frequency series (Fourier transform of the signal). This information is then extracted into a certain matrix with a fixed structure and the SVs of that matrix are sought. This transform can be used as a preprocessing stage in pattern clustering methods. The results in using it indicate that the performance of a combined system including this transform and classifiers is comparable with the performance of using other feature extraction methods such as wavelet transforms. To evaluate TFM-SVD, we applied this new method and artificial neural networks (ANNs) for ballistocardiogram (BCG) data clustering to look for probable heart disease of six test subjects. BCG from the test subjects was recorded using a chair-like ballistocardiograph, developed in our project. This kind of device combined with automated recording and analysis would be suitable for use in many places, such as home, office, and so forth. The results show that the method has high performance and it is almost insensitive to BCG waveform latency or nonlinear disturbance.
\end{abstract}

Copyright (C) 2007 Alireza Akhbardeh et al. This is an open access article distributed under the Creative Commons Attribution License, which permits unrestricted use, distribution, and reproduction in any medium, provided the original work is properly cited.

\section{INTRODUCTION}

Ballistocardiogram (BCG) $[1,2]$ is a movement-related signal caused by shifts in the center of the mass of the blood, which consists of components attributable to cardiac activity, respiration, and body movements. One of the advantages of the BCG measurement is that no electrodes are needed to be attached to the subject [3]. Therefore, it could provide the possibility of serving as a relatively low-cost, noninvasive, easy-to-use, home screening procedure for cardiac performance assessment. Also, the BCG provides complimentary information to traditional ECG measurements, telling us more about the mechanical properties of the heart. An example of the BCG signal is shown in Figure 1 [4]. During the past several years, some classical as well as intelligent pattern recognition methods have been developed for BCG analysis. Yu and Dent [3] and Jansen et al. [5] listed some of these methods in more detail. The performance of some of the existing methods is very good when not considering electromechanical drifts, BCG cycle's latency, motion artifacts, or other kinds of nonlinear disturbances [6]. The results will have some errors if such important factors are not counted. Another important limitation of the existing approaches is their suitability for fast implementation as well as online processing.

In this paper, we introduce a new method for feature extraction, which we call "time-frequency moments singular value decomposition (TFM-SVD)." To evaluate the ability of the new method, we used it to compute the most important BCG waveform features and then clustered them using an artificial neural network (ANN). To find the performance of the combined approach, six test subjects from three groups were used: two healthy young persons, two healthy old men, and two old men with a previous heart 


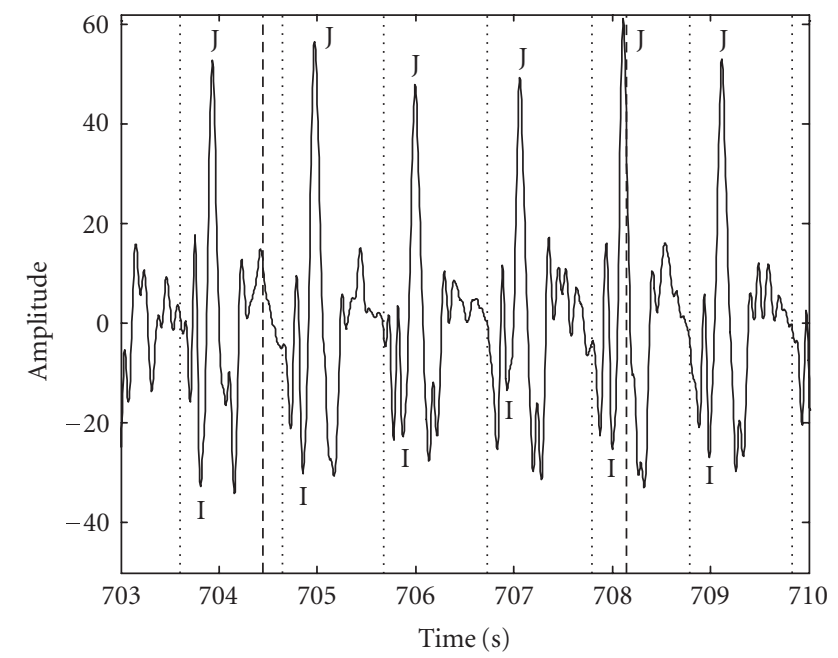

FIGURE 1: The heart-originated BCG signal during one respiratory cycle [4]. The "I" and "J" spikes are marked with circles. The dotted lines point the " $\mathrm{R}$ " spikes detected from the ECG signal, and the dashed lines point the beginning and the end of the respiratory cycle.

(myocardial) infarction. In Section 2, the used measurement system is presented. The developed signal processing methods are presented in Section 3, followed by results and discussion.

\section{MEASUREMENT SYSTEM}

In the past, the BCG has been measured using several different methods. The most traditional way is to allow free body movements in a supine (lying) position and to measure the body movements in the horizontal direction (headto-toe axis). In our work, we use pressure sensitive $\mathrm{EMFi}^{1}$ sensors $[7,8]$ to measure small forces generated by the body in a sitting position. For this purpose, a special measurement chair fitted with EMFi-sensors under the upholstery was constructed [9]. Two sensor films were used, one for the seat and one for the backrest. In this paper, we only use the signal recorded from the seat sensor. The charge signal from the EMFi-sensors is converted to a voltage signal using a smallcharge amplifier. The converted signal was recorded together with electrocardiogram (ECG), the impedance cardiogram (ICG), and some other biosignals using a commercial CircMon (JR Medical Ltd, Tallinn, Estonia) circulation monitor with a sampling frequency of $200 \mathrm{~Hz}$. The sampling rate is fixed in CircMon, but according to Shannon theorem and BCG bandwidth, the sampling rate of $200 \mathrm{~Hz}$ is adequate, it is about five times higher than BCG bandwidth $(0-40 \mathrm{~Hz})$ [10]. The chair and other devices of the system can be seen in Figure 2. More information about the use of EMFi-sensors for BCG measurement and the performance of the measurement system can be found from publications $[4,11]$.

\footnotetext{
${ }^{1}$ EMFi is a registered trademark of Emfit Ltd.
}

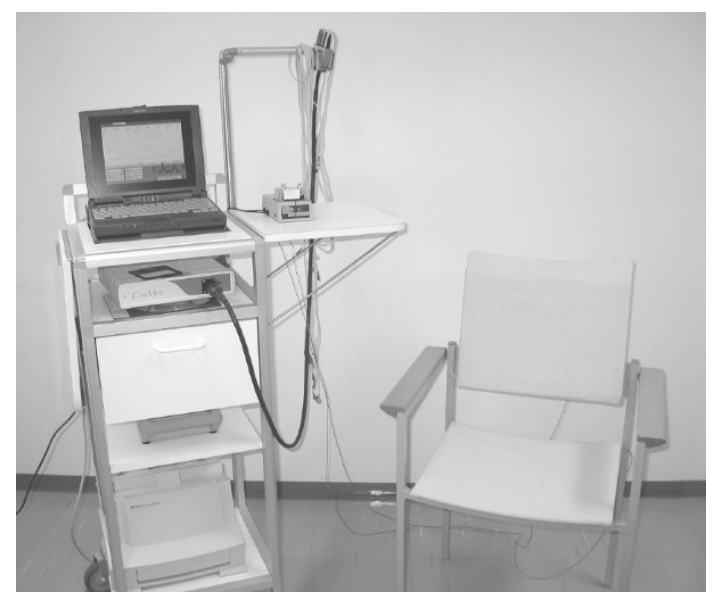

FIgUre 2: A chair equipped with an EMFi-film sensor, the charge amplifier, a CircMon device, and a laptop PC.

Another similar BCG measurement technique is the static-charge sensitive bed (SCSB). Its structure and operation are more complex, as it measures the "rubbing" action applied to the film. Due to its structure, SCSB is more capable in detecting movement also parallel to the film plane [12]. The EMFi sensor is closer to a traditional force sensor, which measures the force applied vertical to the film [13]. According to our experience, the signals measured from a supine subject have similar temporal and spectral properties, but the results are not the same. Therefore, in this application, with measurements carried out in a sitting position and with the sensor directly under the subject's body, the EMFi-sensor is well suited.

\subsection{Patient recordings}

BCG of the test subjects was recorded in a clinical trial at Tampere University Hospital in 2004-2005 using the measurement hardware presented in this section. A reference ECG signal was recorded simultaneously from the chest of the subject's body. Examples of the recorded BCG/ECG signals are presented in Figure 3.

\section{SIGNAL ANALYSIS PROCEDURES}

Signal processing methods were applied to the data after the recordings. The procedure we used for post-recording classification (Figure 4) of the recorded BCG data includes these three steps: (A) BCG cycle extraction using the ECG signal of the subject (segmentation stage); (B) feature computing using TFM-SVD; (C) using ANNs to cluster features. These stages are discussed in detail in the following subsections.

\subsection{BCG data segmentation}

The final aim of this research work is the BCG cycle clustering, not BCG data segmentation. In this work, we used the ECG data of the subjects to extract BCG waveforms in every 


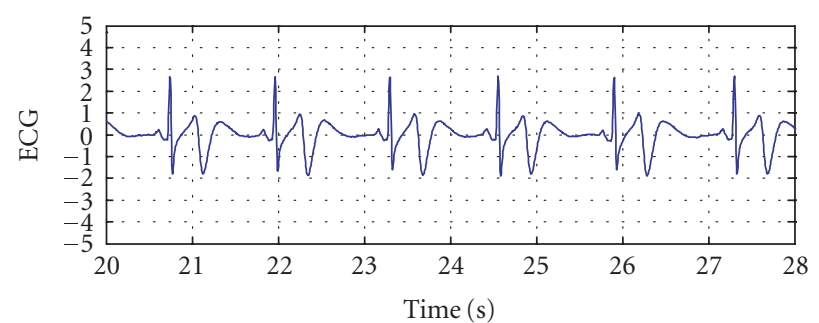

(a)

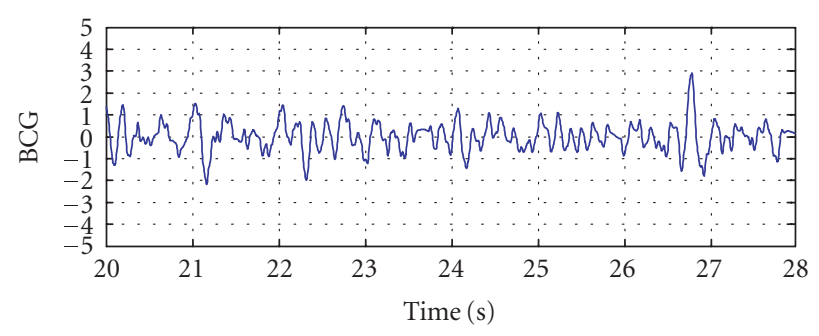

(b)

FIgURE 3: Typical ECG and BCG records of a normal subject.

cardiac period. To achieve this aim, the R-components of the ECG signal, threshold detecting, and 250 point windows were used. The raw signal from the EMFi-sensor includes components of breathing, motion artifacts, low and high frequency noises, and so forth. Thus, we had to use a bandpass filter to extract the heart-originated BCG signal. According to our experience, if we use a bandpass filter of $[3,14] \mathrm{Hz}$ to smooth the recorded BCG signals, we can extract the most important components of BCG signals for pattern recognition purposes, and remove other components. Of course, for monitoring or other purposes, we must use another type of filtering, but for our application this bandwidth was found suitable.

\subsection{BCG features extraction using TFM-SVD}

To define TFM-SVD, first we must be familiar with singular value decomposition (SVD), briefly explained below.

\subsubsection{Singular value decomposition}

Singular value decomposition is used because it captures the essential information of a matrix, somewhat similarly as the eigenvalues do. A singular value and the corresponding singular vectors of a rectangular matrix $A$ are a scalar $\sigma$ and a pair of vectors $u$ and $v$ that satisfy

$$
\begin{gathered}
A \nu=\sigma u, \\
A^{T} u=\sigma \nu .
\end{gathered}
$$

With the singular values on the diagonal of a diagonal matrix and the corresponding singular vectors forming the columns

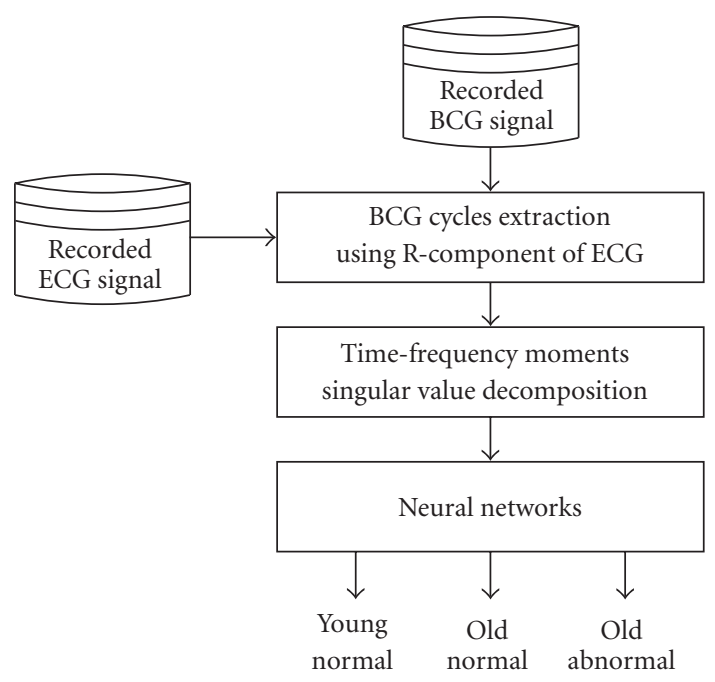

FIGURE 4: Block diagram of the post-recording signal analysis.

of two orthogonal matrices $U$ and $V$, we have

$$
\begin{gathered}
A V=U \sum, \\
A^{T} U=V \sum .
\end{gathered}
$$

Since $U$ and $V$ are orthogonal, this becomes the singular value decomposition

$$
A=U \sum V^{T}
$$

The full singular value decomposition of an $m$-by- $n$ matrix involves an $m$-by- $m U$, an $m$-by- $n \sum$, and an $n$-by- $n V$. In other words, $U$ and $V$ are both square and are the same size as $A$. If $A$ has many more rows than columns, the resulting $U$ can be quite large, but most of its columns are multiplied by zeros in $\sum$. In this situation, the economy sized decomposition saves both time and storage by producing an $m$-by- $n U$, an $n$-by- $n \sum$, and the same $V[15]$.

\subsubsection{Time-frequency moments singular value decomposition}

As explained in the previous section, the singular value decomposition (SVD) is an appropriate tool for analyzing a mapping from one vector space into another vector space, possibly with a different dimension. If the matrix under analysis is square, symmetric, and positive definite, then its eigenvalue and singular value decompositions are the same. In particular, the singular value decomposition of a real matrix is always real, but the eigenvalue decomposition of a real nonsymmetric matrix might be complex [15]. However, if we use it to find SVs of an $m$-by-1 or 1-by- $m$ array with elements representing samples of a signal, it will return only one singular value that is not enough to express all parts of a signal.

To overcome this problem, we introduce a new kind of feature extraction method the so-called time-frequency 
moments singular value decomposition (TFM-SVD) in this paper. In this new method, we suggest the use of eight statistical features of the input signal in time domain as well as frequency domain. The reason for the use of both time and frequency domains is that if the signal under analysis is a nonstationary signal, as most biological signals and our example BCG are, then we will need a time-frequency analyzing tool to find its most important features, and we cannot use the transforms that use either temporal or frequency features. Although there are some other existing transforms such as the wavelet transform to analyze BCG [14, 16-18], we tried to introduce another kind of BCG analysis which is able to give results similar to results of the wavelet transform. Our suggestion is to form two matrices: one to store the most elementary statistical parameters of the signal in time domain $(M t)$ and another one $(M f)$ to store the most elementary statistical parameters of the frequency domain representation of the signal. After that, we can use singular value decompositions (SVD) to extract singular values (SVs) of those matrices. We can use these obtained SVs as the most important features of the input signal. The proposed method has the following steps to compute eight features of signal " $x[n]$ " with length $N$ (samples), which is inside the range $[-5,5]$.

(1) Run fast fourier transform (FFT) algorithm to find spectrum $(y[m])$ of signal $x[n]$.

(2) Compute four statistical moments of time-series $x[n]$ which are

$$
\begin{gathered}
\text { mean }=E[x]=\mu, \\
\text { variance_t }=E\left[(x-\mu)^{2}\right], \\
\text { sharpness_t }=E\left[(x-\mu)^{3}\right], \\
\text { 4th moment_t }=E\left[(x-\mu)^{4}\right] .
\end{gathered}
$$

(3) Compute statistical moments of frequency-series $y[m]:$

$$
\begin{gathered}
\text { mean }=E[y]=\mu f, \\
\text { variance } f_{-}=E\left[(y-\mu f)^{2}\right], \\
\text { sharpness } f_{-}=E\left[(y-\mu f)^{3}\right], \\
\text { 4th moment_f }=E\left[(y-\mu f)^{4}\right] .
\end{gathered}
$$

(4) Assemble a new matrix $(M t)$ with the four following statistical moments of the input signal in time domain $(x[n])$ :

$$
M t=\left[\begin{array}{cc}
a * \mu & \text { variance } \_t / b \\
\text { sharpness_t } t / c & 4 \text { th moment_t } t / d
\end{array}\right],
$$

where $a, b, c, d$ are constant values and must be in the range where four elements of this matrix are scaled to the range $[L 1, L 2]$. This scaling is needed to make all elements of the matrix $M t$ uniform to the same range.

(5) Assemble another matrix $(M f)$ with the following four statistical moments of the spectrum of the input

$$
\begin{aligned}
& (y[m]): \\
& M f=\left[\begin{array}{cc}
\text { af } * \mu f & \text { variance }_{-} f / \text { bf } \\
\text { sharpness }_{-} f / \mathrm{cf} & 4 \text { th moment } f / \text { df }
\end{array}\right],
\end{aligned}
$$

where, again, af, bf, cf, df are constant values and must be in the range where four elements of the matrix $M f$ are scaled to the range $[L 3, L 4]$. This scaling is needed to make all elements of the matrix $M t$ uniform to the same range.

(6) Find singular values (SVs) of the matrix $M t$ : SVD $(M t)$; this will return two SVs for any kind of input signal $x[n]$, because the dimension of $M t$ matrix is $2 \times 2$.

(7) Find singular values (SVs) of matrix $M f$ : SVD $(M f)$; this will return another two SVs for any kind of spectrum $(y[m])$ of an input signal, because the dimension of the $M f$ matrix is $2 \times 2$.

(8) Finally, assemble the so-called time-frequency moments (TFM) vector with dimension $4 \times 1$ :

$$
\text { TFM matrix }=\left[\begin{array}{c}
\operatorname{SVD}(M t) \\
\operatorname{SVD}(M f)
\end{array}\right] .
$$

As can be seen, these steps will return fixed four SVs of time-frequency moments for any type of signal with any size. The properties of this new kind of the feature extraction method are suitable especially for our application because we would like to optimize and reduce the dimension of the input signals (BCG cycles) as much as possible. This method is quite insensitive to phase shifting of the BCG cycles because of its structure.

The reason behind choosing the above-mentioned four moments is their ability to extract essential information from both time and frequency domains. In probability theory and statistics, the mean is the expected value of a real-valued random variable ( $\operatorname{signal} x$ ), which is also called the population mean. For a data set, the mean is just the sum of all the observations divided by the number of observations. The variance of a random variable is a measure of its statistical dispersion, indicating how far from the expected value its values typically are. The variance of a real-valued random variable is its second central moment, and it also happens to be its second cumulant. The variance of a random variable is the square of its standard deviation. Sharpness shows the amplitude and the direction of the changes in the random process. The fourth moment, similar to Kurtosis, is a measure of the "peakedness" of the probability distribution of a real-valued random variable. Higher fourth moment means that more of the variance is due to infrequent extreme deviations, as opposed to frequent modestly sized deviations. The other four features indicate the same measures that form the frequency domain of a real-valued random variable. In our tests, we found that higher than four moments do not give us new information compared to moments one to four. Although we recommend only using the first four moments of a real-valued random variable in the proposed algorithm, it is possible to add more moments, in both time and frequency domains, to the abovementioned algorithm. 
Singular value decomposition is used because it captures the essential information of a matrix (compression), somewhat similarly as the eigenvalues do. Referring to our experiences, using SVD after the extraction of the moments gives us better ability to train a neural network for pattern classification.

The choice of TFM normalization parameters depends on the input signal range. For instance, if the input signal is inside the range $\left[\alpha_{1}, \alpha_{2}\right]$ where $\alpha_{2}>\alpha_{1}$, the time domain moments will be in these ranges:

$$
\begin{aligned}
& \alpha_{1} \leq \mu \leq \alpha_{2}, \\
& 0 \leq \text { variance } \_t t\left(\alpha_{2}-\alpha_{1}\right)^{2} \text {, } \\
& -\left(\alpha_{2}-\alpha_{1}\right)^{3} \leq \text { sharpness } \_t \leq\left(\alpha_{2}-\alpha_{1}\right)^{3} \text {, } \\
& 0 \leq 4 \text { th moment_ } t \leq\left(\alpha_{2}-\alpha_{1}\right)^{4} \text {. }
\end{aligned}
$$

So, the constant values of time domain must be

$$
\begin{aligned}
& a=\frac{\alpha_{2}-\alpha_{1}}{\max \left(\left|\alpha_{1}\right|,\left|\alpha_{2}\right|\right)} \\
& b=\alpha_{2}-\alpha_{1} \\
& c=\left(\alpha_{2}-\alpha_{1}\right)^{2} \\
& d=\left(\alpha_{2}-\alpha_{1}\right)^{3}
\end{aligned}
$$

to have an $M t$ matrix in the range

$$
\left[L 1=-\left(\alpha_{2}-\alpha_{1}\right), L 2=+\left(\alpha_{2}-\alpha_{1}\right)\right]
$$

The moments in the frequency domain will be in these ranges:

$$
\begin{gathered}
N * \alpha_{1} \leq \mu_{f} \leq N * \alpha_{2}, \\
0 \leq \text { variance }_{-} f \leq N^{2} *\left(\alpha_{2}-\alpha_{1}\right)^{2}, \\
-N^{3} *\left(\alpha_{2}-\alpha_{1}\right)^{3} \leq \text { sharpness }_{-} f \leq N^{3} *\left(\alpha_{2}-\alpha_{1}\right)^{3}, \\
0 \leq 4 \text { th moment } f \leq N^{4} *\left(\alpha_{2}-\alpha_{1}\right)^{4},
\end{gathered}
$$

where $N$ is the length of the signal. Thus, the constant values of frequency domain must be

$$
\begin{aligned}
& \mathrm{af}=\frac{\alpha_{2}-\alpha_{1}}{N * \max \left(\left|\alpha_{1}\right|,\left|\alpha_{2}\right|\right)}, \\
& \mathrm{bf}=N^{2} *\left(\alpha_{2}-\alpha_{1}\right), \\
& \mathrm{cf}=N^{3} *\left(\alpha_{2}-\alpha_{1}\right)^{2}, \\
& \mathrm{df}=N^{4} *\left(\alpha_{2}-\alpha_{1}\right)^{3}
\end{aligned}
$$

to have an $M f$ matrix in the range

$$
\left[L 3=-\left(\alpha_{2}-\alpha_{1}\right), L 4=+\left(\alpha_{2}-\alpha_{1}\right)\right] .
$$

\subsubsection{BCG feature computing using TFM-SVD}

In this study, we used TFM-SVD and computed TFM-SVs of every BCG cycle $x[n]$ using the procedure explained in Section 3.2.2. Every BCG cycle has 250 samples because of our measurement system and its sampling rate $(200 \mathrm{HZ})$. The TFM-SVD method helps us to find the most important features of the BCG cycles (waveforms) and to reduce the cycle's dimension $(N)$ from $N=250$ features to 4 features. Before using the TFM-SVD algorithm, we must initialize its constant values. In this study, we used $N=250$, $L 1=L 3=-10$, and $L 2=L 4=10$, so constants are $a=2, b=10, c=100, d=1000$, af $=2 / 250$, bf $=$ $250^{2} * 10=6.25 * 10^{5}, \mathrm{cf}=250^{3} * 10^{2}=1.5625 * 10^{9}$, df $=250^{4} * 10^{3}=3.90625 * 10^{12}$. On the other hand, they shift $M t$ and $M f$ elements' values to the range $[-10,10]$ if the input signal's values $(x[n])$ are inside the range $[-5,5]$. This range $(L 1=L 3=-10$, and $L 2=L 4=10)$ is chosen for convenience and there are no restrictions on initializing the TFM-SVD constants. The results (TFM-SV features of whole extracted BCG cycles) in a 3D presentation for six typical subjects (two young normal, two old normal, and two old abnormal subjects) are shown in Figure 5. As we explained, the TFM-SVD algorithm returns only four features for every BCG cycle. To show how these features are scattered in a $3 \mathrm{D}$ presentation, we have to consider only the first three features and ignore the fourth one for every BCG cycle.

\subsection{BCG data clustering using artificial neural networks}

For BCG feature classification, we used two kinds of very famous ANNs: multilayer perceptrons (MLPs) and radial basis functions (RBFs) $[19,20]$. Before presenting the TFM-SVD and the coefficients of the BCG cycles to the neural network, we must normalize them to the range $[0,1]$.

\subsubsection{Multilayer perceptrons}

Multilayer perceptrons (MLPs) are feed-forward neural networks trained with the standard back propagation algorithm. They are supervised networks, which means that they require a desired response in order to be trained. They learn how to transform input data into a desired response, and therefore they are widely used for pattern classification. With one or two hidden layers, they can approximate virtually any inputoutput map. They have been shown to approximate the performance of optimal statistical classifiers in difficult problems. Most neural network applications involve MLPs.

\subsubsection{Radial basis functions}

RBF networks $[19,20]$ employ neurons that consist of radial basis functions. In contrast to classic multilayer perceptrons, the activation of a neuron is not given by the weighted sum 


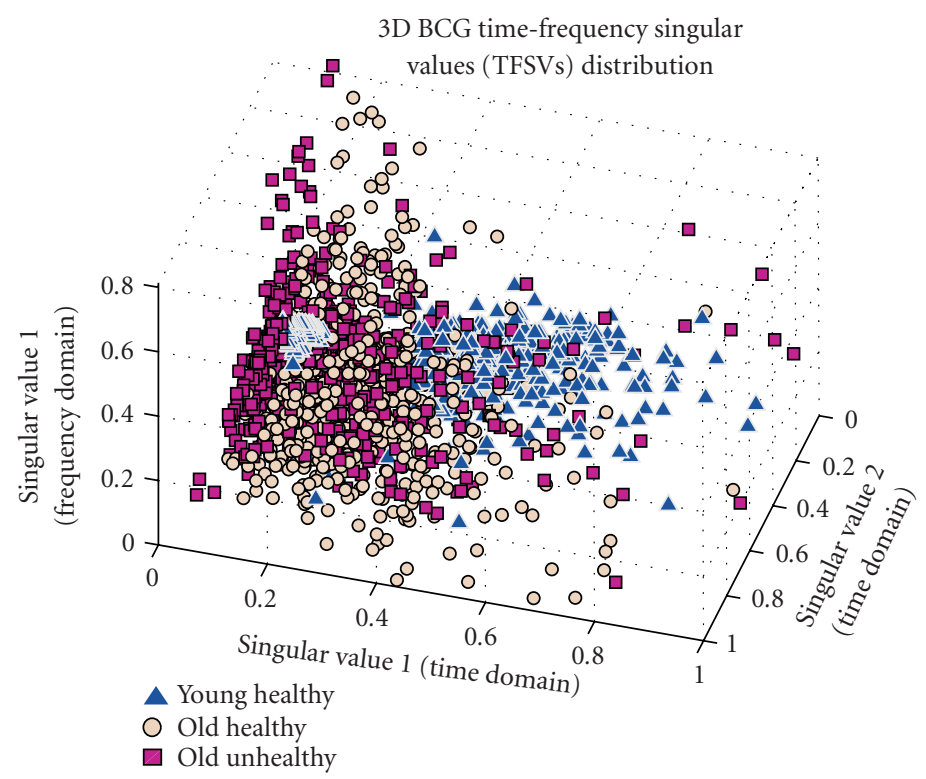

FIGURE 5: A 3D scattergram for TFM-SV features of all extracted BCG cycles of six subjects under test (two young normal, two old normal, and two old abnormal subjects). Only the first three features of the TFM-SVD algorithm are normalized to [0,1] and shown in this $3 \mathrm{D}$ visualization.

of all its inputs but by the computation of a radial basis function. Generally, the kernels-Gaussian function

$$
\varphi\left(u ; t_{k}\right)=\exp \left(-\frac{1}{\sigma_{k}^{2}}\left\|u-t_{k}\right\|^{2}\right), \quad k=1,2, \ldots, k, \sigma_{k}>0
$$

is used where $u$ is the input of the neuron, $t$ is the basis of the neuron, and $\sigma$ is the amplitude of the neuron. RBF networks are feed-forward networks and consist of one input layer $(u)$, one hidden layer of Gaussian neurons $(H)$, and one output layer $(y)$. The value of an output unit $y_{i}$ (given a network input $u$ ) is computed by

$$
y_{i}=\sum_{k=1}^{K} w_{i k} \varphi_{i}\left(u ; t_{k}\right)+w_{i 0}
$$

where $w_{i k}$ is the weight (= height) of neuron Hi for output $y_{i}$ and $w_{i 0}$ is a general threshold (bias) of output $y_{i}$ subtracted from the weighted inputs. The Gaussian neurons work as experts for certain areas of the d-dimensional input space. Activation of each neuron depends on its distance to the input vector. Learning algorithms like back propagation (we used a stochastic learning $[9,10])$ can be used to adjust the nonfixed parameters of the network including kernels centers, weights, and stigma.

\section{RESULTS}

To demonstrate the performance of our approaches and to compare results, we used MLP (two hidden layers with 15 and 10 neurons relatively) and RBF neural networks (hidden layer with 15 neurons) with 4 inputs, and 3 outputs to classify 6 subjects into 3 categories: young healthy students aged between 20-30 years ( 2 subjects), old healthy men aged between 50-70 years ( 2 subjects), and two old subjects (50-70 years old) with a heart infarction in their medical history. The number and the size of the hidden layers for both MLP and RBF networks were optimized for this application (BCG classification). If we use another kind of physiological signal or change the number of subjects under test, we must check the structure of the network again.

For every subject of the three categories, the previous stage (TFM-SVD) gave us four features of every BCG cycle. These data were normalized, mapped to the area $[0,1]$, and finally saved randomly into a unique data matrix. We used a small part of the data for training artificial neural networks (500 BCG cycles used for MLP nets and 300 BCG for RBF nets) and the rest of the data (2000 BCG cycles) for testing the performance of the ANN classifier, not using the same data for training and testing the system. On the other hand, in this study there were no excluded subjects for testing and we used the same subjects for both training and testing the MLP and RBF neural networks. However, to develop a complete diagnosing system to find the heart condition of the subjects under test, we had to exclude some subjects for testing its performance.

Table 1 shows the performance of the two approaches. In this test, the MLP performed clearly better than the RBF network (SBJs in the table means subjects). If the MLP had made the decision to which class the subject belongs, it would have classified every subject correctly. This means that more than $50 \%$ of the BCG cycles for every subject were always in the right class when BCG cycles were selected randomly. Thus we can say that the whole recordings were classified correctly 
TABLE 1: Results of the BCG classification using neural networks and BCG cycles in Figure 5. TFM-SVD is used for computing BCG waveform features. SBJ means subject. Every cell shows the correct classification percentage for every class. Class 1: young healthy students aged between 20-30 years (2 subjects). Class 2: old healthy men aged between $50-70$ years (2 subjects). Class 3: two old subjects (50-70 years old) with a previous heart infarction. Overall (\%): performance computed using randomly selected training and testing BCG data of 6 subjects.

(a) MLP neural network using 500 epochs for training and 2000 for testing net

\begin{tabular}{|c|c|c|c|c|c|}
\hline & & Class1 & Class2 & Class3 & Overall \\
\hline \multirow{2}{*}{ Class 1} & SBJ1 & $97 \%$ & $3 \%$ & - & \\
\hline & SBJ2 & $93 \%$ & $7 \%$ & - & \\
\hline \multirow{2}{*}{ Class 2} & SBJ1 & - & $93 \%$ & $7 \%$ & \\
\hline & SBJ2 & - & $68 \%$ & $32 \%$ & \\
\hline \multirow{2}{*}{ Class 3} & SBJ1 & - & $42 \%$ & $58 \%$ & \\
\hline & SBJ2 & - & $7 \%$ & $91 \%$ & \\
\hline \multicolumn{5}{|l|}{ Overall } & $85 \%$ \\
\hline
\end{tabular}

(b) RBF neural network using 300 epochs for training and 2000 for testing net

\begin{tabular}{|l|c|c|c|c|c|}
\hline \multicolumn{2}{|c|}{} & Class 1 & Class 2 & Class 3 & Overall \\
\hline \multirow{2}{*}{ Class 1 } & SBJ1 & $\mathbf{8 3} \%$ & $17 \%$ & - & \\
\cline { 2 - 5 } & SBJ2 & $\mathbf{9 0} \%$ & $10 \%$ & - & \\
\hline \multirow{2}{*}{ Class 2 } & SBJ1 & - & $\mathbf{6 7} \%$ & $33 \%$ & \\
\cline { 2 - 5 } & SBJ2 & - & $\mathbf{8 4} \%$ & $16 \%$ & \multirow{2}{*}{} \\
\hline \multirow{2}{*}{ Class 3 } & SBJ1 & - & $100 \%$ & $\mathbf{0} \%$ & $\mathbf{7 3} \%$ \\
\cline { 2 - 5 } & SBJ2 & - & $49 \%$ & $\mathbf{5 1} \%$ & \\
\hline \multicolumn{2}{|l|}{ Overall } & & & \\
\hline
\end{tabular}

using the MLP network. This means that the local minima or nonlinear disturbances have only a small effect on the MLP's overall performance. In terms of our investigations, this performance will not increase if more epochs or adaptation cycles are used during the training phase for the MLP network.

RBF would have misclassified one subject and would have nearly misclassified another. Again, in terms of our research, the results will not improve if more epochs or adaptation cycles are used during the training phase for the RBF network.

If we compare the results of using the TFM-SVD method for BCG's feature extraction with our results using the wavelet transforms (WT) to extract the most important features of the BCG cycles [14, 16-18], we see that the results for both of these methods are comparable. The overall performance of the MLP network using WT was above $90 \%$, while it was $85 \%$ using TFM-SVD for the same (six) subjects under test. Although the performance using the TFM-SVD was more than $5 \%$ lower than using the WT, the TFM-SVD is faster and easier to implement than the WT. This is because the WT is a multiresolution time-frequency transform and we must use an iterative algorithm, the so-called Mallat algorithm, to find a higher resolution in the frequency domain and low resolution in the time domain [21]. For instance, in $[14,16-18]$, we used wavelet coefficients of BCG cycles in level (resolution) six of Mallat algorithm and this needed some time to compute.

\section{DISCUSSION}

To discriminate BCG features, researchers have presented different methods $[1,3]$. Most of the existing methods have high accuracy in the BCG features discrimination, while not taking into consideration that the BCG waveforms have latency or nonlinear disturbances such as motion artifacts and electromechanical drifts/noises. However, ignoring these kinds of important issues may potentially give us incorrect information about patients.

In this paper, we developed approaches which have good performance, even with nonlinear disturbances or latency. To overcome these kinds of phenomena, we introduced a new feature extraction method that we call "time-frequency moments singular value decomposition (TFM-SVD)." For the classification of the extracted BCG cycles, we used two neural classifiers, MLP and RBF nets. The results showed that this classifier multilayer network has a high performance, even with nonlinear disturbance or latency. The MLP had a better performance compared to the RBF. Because of the local minima phenomena, the RBF could not classify class 3 (old abnormal men) well. This inability will increase if we use more than 300 BCG cycles for training the RBF net and more than 500 for the MLP net (overtraining problem).

Classifying the BCG cycles correctly is very important for post processing. The first stage of our classification system is a segmentation stage used to extract BCG cycles. We currently use the R-components of the ECG signal for the detection of the cardiac period, but it is also possible to do the extraction without the ECG. We have already developed the BCG segmentation method without using ECG $[15,16]$. The method uses a bandpass-filtered low-frequency coarse BCG signal for the detection of the I-component of BCG, and it is used for the detection of the cardiac period. It should also be mentioned that the developed method in this paper is not limited to BCG data classification and it can be used to other applications of physiological signal processing such as evoke potentials, EEG, EOG, and EMG.

Our initial aim in this study is only to introduce a system to classify BCG waveforms. To have a complete diagnosing system, we need much more subjects from all of the three categories, which takes time and more investigations. The more developed system and analysis method could be used for the automatic measurement and evaluation of a person's health and heart condition, when he/she is visiting a doctor's office. The automated analysis assists the doctor in faster decision making and directs the doctor to perform needed additional measurements. The system could also be used in home health monitoring and long term follow-up monitoring applications.

\section{ACKNOWLEDGMENTS}

The authors would like to thank Dr. Tiit Kööbi and Dr. Väinö Turjanmaa from Tampere University Hospital for their involvement in the development of the measurement 
system hardware and organizing the test measurements in Tampere University Hospital. We also thank Ms. Marjaana Ylhäinen and Mrs. Pirjo Järventausta for carrying out the measurements, and all the test subjects for their participation. Finally, we would like to thank Mr. John Shepherd from Tampere University of Technology Language Center for proofreading this article. This study was financially supported by the Academy of Finland, the Proactive Information Technology Program 2002-2005, and the Finnish Center of Excellence Program 2000-2005.

\section{REFERENCES}

[1] I. Starr, "Further clinical studies with the ballistocardiograph on abnormal form, on digitalis action, in thyroid disease, and in coronary heart disease," Transactions of the Association of American Physicians, vol. 59, pp. 180-189, 1946.

[2] B. M. Baker Jr., W. R. Scarborough, R. E. Mason, et al., "Coronary artery disease studied by ballistocardiography: a comparison of abnormal ballistocardiograms and electrocardiograms," Transactions of the American Clinical and Climatological Association, vol. 62, p. 191, 1950.

[3] X. Yu and D. Dent, "Neural networks in ballistocardiography (BCG) using FPGAs," in IEE Colloquium on Software Support and CAD Techniques for FPGAs, pp. 7/1-7/5, London, UK, April 1994.

[4] M. Koivuluoma, L. C. Barna, and A. Värri, "Signal processing in ProHeMon project: objectives and first results," in Proceedings of the Proactive Computing Workshop (PROW'04), pp. 55-58, Helsinki, Finland, November 2004.

[5] B. H. Jansen, B. H. Larson, and K. Shankar, "Monitoring of the ballistocardiogram with the static charge sensitive bed," IEEE Transactions on Biomedical Engineering, vol. 38, no. 8, pp. 748751, 1991.

[6] A. Akhbardeh, M. Farrokhi, and A. V. Tehrani, "EEG features extraction using neuro-fuzzy systems and shift-invariant wavelet transforms for epileptic seizures diagnosing," in Proceedings of 26th Annual International Conference of the Engineering in Medicine and Biology Society (EMBC'04), vol. 1, pp. 498-502, San Francisco, Calif, USA, September 2004.

[7] J. Lekkala and M. Paajanen, "EMFi-new electret material for sensors and actuators," in Proceedings of 10th International Symposium on Electrets (ISE '99), pp. 743-746, Delphi, Greece, September 1999.

[8] M. Koivuluoma, J. Alametsä, and A. Värri, "EMFI as physiological signal sensor, first result in ProHeMon project," in Proceedings of the URSI XXVI Convention on Radio Science and Second Finnish Wireless Communication Workshop, p. 2s, Tampere, Finland, 2004.

[9] S. Junnila, T. Koivistoinen, T. Kööbi, J. Niitylahti, and A. Värri, "A simple method for measuring and recording ballistocardiogram," in Proceedings of 17th Biennial International EURASIP Conference (BIOSIGNAL '04), pp. 232-234, Brno, Czech Republic, June 2004.

[10] P. Strong, Biophysical Measurements, Tektronix, Beaverton, Ore, USA, 1970.

[11] S. Junnila, A. Akhbardeh, T. Koivistoinen, and A. Värri, "An EMFi-film sensor based Ballistocardiographic chair: performance and cycle extraction method," in Proceedings of IEEE Workshop on Signal Processing Systems (SiPS '05), pp. 373-377, Athens, Greece, November 2005.
[12] J. Alihanka, K. Vaahtoranta, and S.-E. Björkqvist, "Apparatus in medicine for the monitoring and or recording of the body movements of a person on a bed, for instance of a patient," March 1982, US patent no. 4320766.

[13] K. Kirjavainen, "Electromechanical film and procedure for manufacturing same," 1987, US patent no. 4654546.

[14] A. Akhbardeh, M. Koivuluoma, T. Koivistoinen, and A. Värri, "Ballistocardiogram diagnosis using neural networks and shift-invariant daubechies wavelet transform," in Proceedings of 13th European Signal Processing Conference (EUSIPCO '05), p. 4, Antalya, Turkey, September 2005.

[15] S. Haykin, Adaptive Filter Theory, Prentice Hall, Englewood Cliffs, NJ, USA, 1996.

[16] A. Akhbardeh, S. Junnila, T. Koivistoinen, and A. Värri, "Ballistocardiogram classification using a novel transform socalled AliMap and biorthogonal wavelets," in Proceedings of IEEE International Workshop on Intelligent Signal Processing (WISP '05), pp. 64-69, Faro, Portuga, September 2005.

[17] A. Akhbardeh, M. Koivuluoma, T. Koivistoinen, and A. Värri, "BCG data discrimination using daubechies compactly supported wavelet transform and neural networks towards heart disease diagnosing," in Proceedings of the IEEE International Symposium on Intelligent Control, 13th Mediterrean Conference on Control and Automationnl, vol. 2005, pp. 1452-1457, Limassol, Cyprus, June 2005.

[18] A. Akhbardeh, S. Junnila, M. Koivuluoma, T. Koivistoinen, and A. Värri, "Heart disease diagnosing mechatronics based on static charge sensitive chair's measurement, biorthogonal wavelets and neural classifiers," in Proceedings of IEEE/ASME International Conference on Advanced Intelligent Mechatronics (AIM '05), pp. 676-681, Monterey, Calif, USA, July 2005.

[19] A. Akhbardeh and A. Erfanian, "Eye tracking user interface using EOG signal and neuro-fuzzy systems for human-computer interaction aids," M.Sc. thesis, Iran University of Science \& Technoloy, Narmak, Tehran, Iran, 2001.

[20] S. Haykin, Neural Networks: A Comprehensive Foundation, Macmillan College, New York, NY, USA, 1984.

[21] S. Mallat, A Wavelet Tour of Signal Processing, Academic Press, New York, NY, USA, 1997.

Alireza Akhbardeh was born in 1974 and grew up in Tabriz, East Azerbaijan, Iran. He received his B.S. degree in electrical engineering from the Tabriz University in 1998. In 2001, he completed his M.S. degree in electrical engineering-bioelectrics at the Iran University of Science and Technology, Tehran. From 2001 to 2004, he was a Lecturer, an Academic Member, at the Tabriz Azad University and some other universities

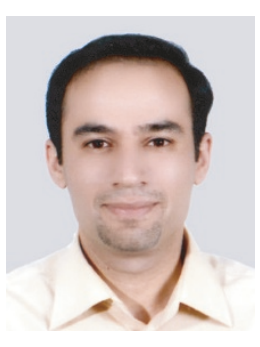
in Iran. At the same time, he was an Instrumentation and Control (I\&C) Specialist in the Ministry of Energy, Tehran, Iran. From 2005, he has been a Research Scientist working towards his Doctoral degree at the Institute of Signal Processing, Tampere University of Technology, Finland. He has been awarded/granted six times during his B.S., M.S., and Ph.D. studies from the universities and the Ministry of Energy of Iran. He also received the Best Presentation Prize in a session of the 2005 IEEE ISIC conference, Cyprus. Between 2004 and 2006, he published more than 30 papers on pattern recognition, development of fast learning algorithms, signal processing, and biomedical applications. 
Sakari Junnila was born in Uusikaupunki, Finland, in 1975. He received his M.S. degree in information technology-digital and computer engineering from the Tampere University of Technology in 1999. Since then, he has worked as a Research Scientist in the Institute of Digital and Computer Systems (2000-2005) and the Institute of Signal Processing from 2005 till now, Tampere University of Technology, Finland. He

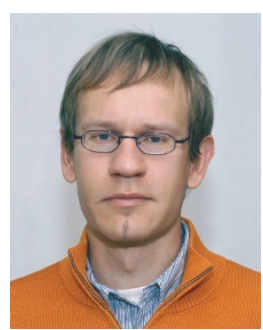
is currently working towards his Dr.Tech. degree in digital and computer engineering at the Tampere University of Technology. His current research interests include wireless short-range communication, medical monitoring and data-acquisition systems, and medical device standardization.

Mikko Koivuluoma was born in Kurikka, Finland, in 1967. He received his M.S. degree in electrical engineering - signal processing from the Tampere University of Technology in 1997. Since then, he has worked as a Research Scientist (1997-1999) and as an Assistant from 2000 till now in the Institute of Signal Processing, Tampere University of Technology, Finland. He is currently working towards his Dr.Tech. degree

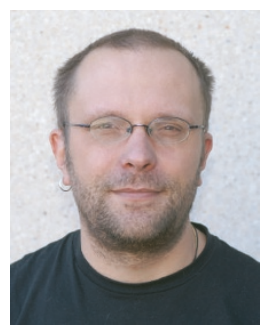
in signal processing at the Tampere University of Technology. His current research interests include ballistocardiographic signals and medical monitoring.

Teemu Koivistoinen was born in Varkaus, Finland, in 1979. He received his M.S. degree in electrical engineering-biomedical engineering from the Tampere University of Technology in 2003. From 2002 to 2006, he worked as a Researcher in the Department of Clinical Physiology, Tampere University Hospital, Finland. He is currently working towards his M.D. and Ph.D. degrees in medicine at the Tampere University. His current research interests include patient monitoring solutions and impedance cardiography solutions.

Alpo Värri received the M.S. degree in electrical engineering in 1986 and the Dr.Tech. degree in signal processing in 1992, both from Tampere University of Technology, Finland. Currently he is a Senior Researcher and the Vice Head of the Institute of Signal Processing of Tampere University of Technology. His research interests include biomedical signal processing and pattern recognition. Since 1994, he has participated

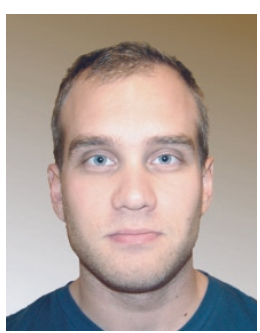
in Health Informatics Standardization within CEN/TC251 and ISO/TC215.

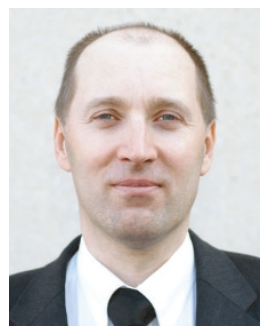

